\title{
Appropriate Use of Bifactor Analysis in Psychopathology Research: Appreciating Benefits and Limitations
}

\author{
Marina A. Bornovalova, Alexandria M. Choate, Haya Fatimah, \\ Karl J. Petersen, and Brenton M. Wiernik
}

\section{Abstract}

Co-occurrence of psychiatric disorders is welldocumented. Recent quantitative efforts have moved toward an understanding of this phenomenon, with the 'general psychopathology' or p-factor model emerging as the most prominent characterization. Over the past decade, bifactor model analysis has become increasingly popular as a statistical approach to describe common/shared and unique elements in psychopathology. However, recent work has highlighted potential problems with common approaches to evaluating and interpreting bifactor models. Here, we argue that, when properly applied and interpreted, bifactor models can be useful for answering some important questions in psychology and psychiatry research. We review problems with evaluating bifactor models based on global model fit statistics. We then describe more valid approaches to evaluating bifactor models and highlight three types of research questions for which bifactor models are wellsuited to answer. We also discuss the utility and limits of bifactor applications in genetic and neurobiological research. We close by comparing advantages and disadvantages of bifactor models to other analytic approaches and noting that no statistical model is a panacea to rectify limitations of the research design used to gather data.

\footnotetext{
1 Sometimes, group factors are called "specific factors." However, "specific factor" more correctly refers to an item's reliable (non-error) variance that is not shared with other items (5).
}

Comorbidity among heterotypic mental disorders is ubiquitous (1), leading some to suggest mental disorders have more common/shared than unique processes. Psychology and psychiatry thus have increasingly used quantitative methods to model covariation among disorders and organize them into higher-order domains (2-4). Such models can separate psychopathology deficits shared by multiple disorders from those unique to specific disorders. One increasingly popular quantitative framework is the bifactor model (5). This model specifies that covariance among observed indicators can be accounted for by a latent general factor, reflecting common variance among all indicators, and one or more latent group factors, ${ }^{1}$ reflecting additional common variance for subsets of indicators $(5,6)$. Group factors are specified to be orthogonal (uncorrelated) to the general factor, so group factors reflect common variance among indicator subsets that is separable from the general factor. ${ }^{2}$

\footnotetext{
2 Several variations on the bifactor model exist, including a bifactor model with correlated group factors (7) and the $S-1$ model $(8,9)$. Correlating group factors, however, changes the interpretation of the latent variables. For a detailed discussion of bifactor model variations, see (10). The random intercept model is a similar model that is useful for testing hypotheses about artefactual indicator covariation due to idiosyncratic differences in response scale usage (e.g., acquiescence biases) (40).
} 
The bifactor model has become popular in psychology and psychiatry research as a method to (a) model commonality and uniqueness across mental disorders and (b) relate said common and unique factors to putative antecedents (e.g., treatments, genetic/environmental factors, neurobiological substrates, personality traits) and outcomes (e.g., cognitive development, academic performance, distress, self-harm, suicidality) (11-17). Many studies have applied bifactor models to document a 'general psychopathology' or $p$-factor reflecting commonality among all forms of psychopathology, along with several narrower psychopathology group factors, most commonly internalizing (depression, anxiety) ${ }^{3}$, externalizing (antisocial and substance use disorders), and psychosis $(7,11,14,19-22)$. Bifactor and related hierarchical models of psychopathology (23) are being incorporated in emerging frameworks for conceptualizing, studying, and diagnosing psychopathology $(21,24)$. Bifactor models are also applied in other psychology subfields to describe constructs such as cognitive abilities (25), personality traits $(26,27)$, and work interests $(28)$.

However, recent conceptual, methodological, and empirical work has highlighted problems with common approaches to evaluating and interpreting bifactor models. These criticisms include a tendency to overfit, such that the bifactor model is inappropriately favored by fit indices; frequent anomalous results, including small factor loadings and zero or negative group factor variances; instability of the general factor, such that the nature of the general factor changes across samples or indicators; problems with identification; questions regarding interpretation of orthogonal latent factors; and concerns about reification in searches for genetic or biological

\footnotetext{
${ }^{3}$ It is also common to model internalizing content as two group factors of fear (phobias, panic) and distress (depression, generalized anxiety) (18).
}

substrates of the $p$-factor (29-32). Here, we suggest that, properly applied and interpreted, bifactor models can be useful for answering some important questions in psychology and psychiatry research. We briefly review problems with the widespread practice of evaluating bifactor and other structural models based solely on global model fit statistics. We then describe better approaches to evaluating bifactor models and highlight three types of research questions bifactor models are wellsuited to answer. Finally, we compare bifactor models to other analytic approaches, discuss applications in psychobiological research, and note that no statistical model is a panacea for limitations of the data-collection design.

\section{Problematic Interpretation of Bifactor Models: Reliance on Global Model Fit}

The major criticism of the bifactor model is its potential for overfitting (33). A common approach to evaluating structural models is to compare several possible models and then retain the model showing the best overall (global) fit statistics, such as $X^{2}$, CFI/TLI, RMSEA, SRMR, or AIC $(14,15)$. This approach is problematic because global fit statistics can favor the bifactor model even when it is a poor description of the data. The confirmatory bifactor model is extremely flexible. The only major constraint imposed on the data is the group factor to which each item belongs. The exact patterns of items' loadings onto the general and group factors are typically permitted to freely vary. Essentially, the model absorbs as much item variance as possible into the general or group factors. Because of this flexibility, the bifactor model can exhibit good global fit even if the pattern of loadings does not resemble a bifactor structure in any meaningful sense. For example, 


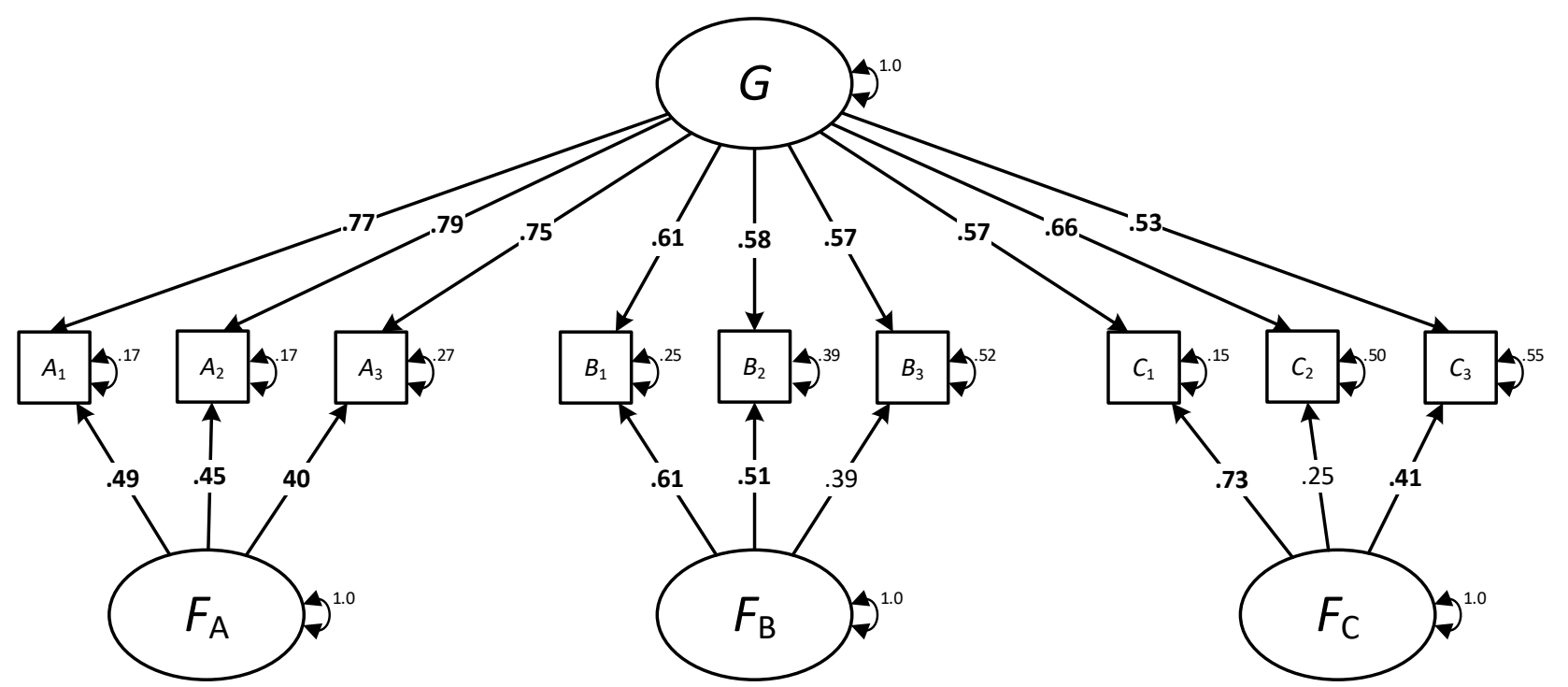

(A) $N=213, \chi^{2}(18)=24.331, \mathrm{TLI}=.988$, RMSEA [90\% Cl] $=.041[.000, .078]$, SRMR [90\% Cl] $=.029[.015, .044]$.

This model shows clear bifactor structure, with good global model fit as well as strong general and group factor loadings.

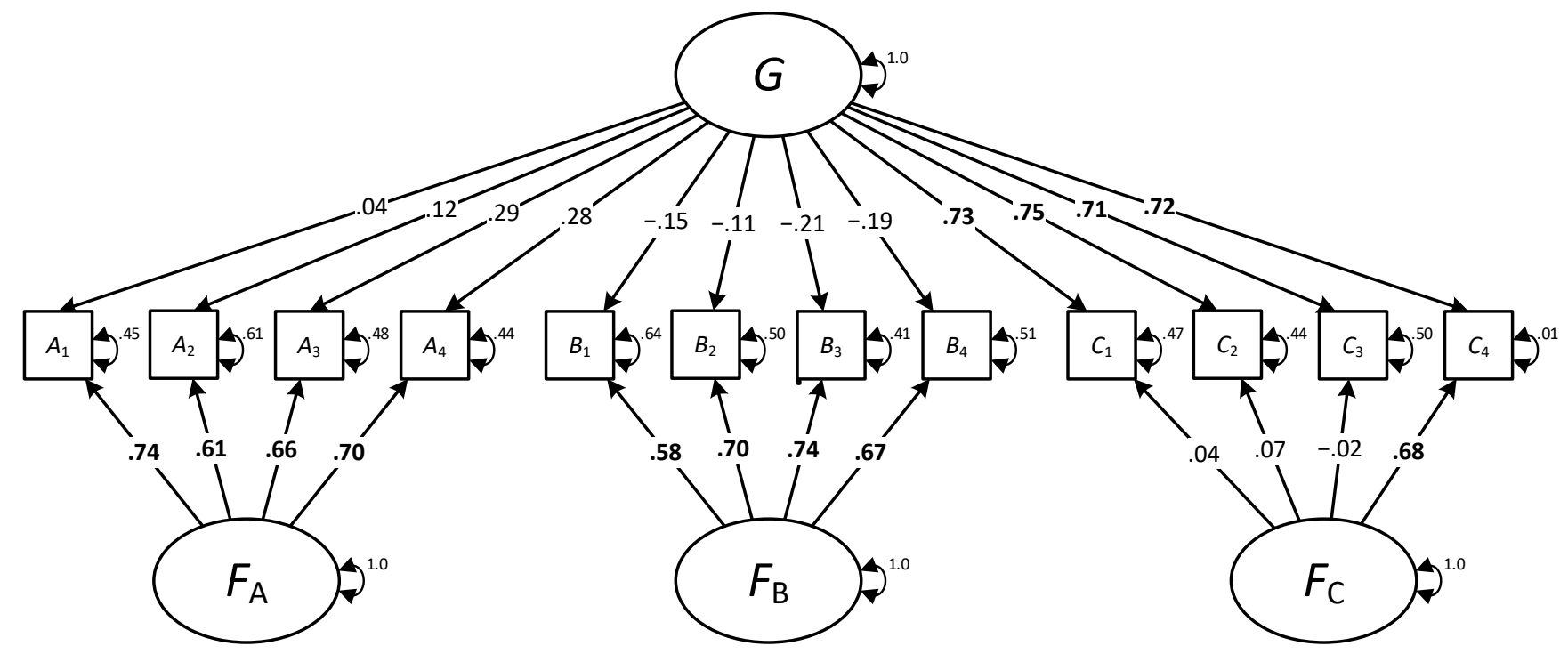

(B) $N=463, \chi^{2}(43)=67.984, \mathrm{TLI}=.978$, RMSEA $[90 \% \mathrm{Cl}]=.035[.018, .051]$, SRMR $[90 \% \mathrm{Cl}]=.042[.024, .059]$. Despite good global model fit, this model does not show clear bifactor structure.

Figure 1. Bifactor models fit to measures of cognitive ability (A) and social attitudes (B). Standardized factor loadings and residual variances. Factor loadings $\geq .40$ in bold. TLI = Tucker- Lewis Index, RMSEA = root mean square error of approximation, $\mathrm{Cl}=$ confidence interval, $\mathrm{SRMR}=$ unbiased standardized root mean square residual. Models fit using the lavaan package (v. 0.6-3) (90) in R (v. 3.5.3) (91).

consider Figure 1B. Here, items from only one subgroup show strong loadings on the general factor, with negligible loadings onto their group factor. Items from the other two subgroups load weakly on the general factor and strongly on their group factors. This 
pattern of loadings implies that the bifactor structure is a poor description of the data, despite adequate global fit statistics. In this example, the general factor is not really a general factor at all, but rather just a group factor that has been shuffled into a different part of the model, cf. (34). In this case, the appropriate conclusion is that the data represent three weakly correlated factors. A similar interpretation can be made in the common case that one of the group factor variances is near-zero or negative (30).

The bifactor model's flexibility can be particularly problematic when comparing bifactor model results across samples (33). Because the model seeks to absorb as much variance as possible, the pattern of factor loadings defining the general or group factors can be unstable across samples $(13,35-37)$. These divergent patterns make comparing results difficult, as the nature and meaning of modeled latent factors is not consistent.

The bifactor model's flexibility can also enable it to show superior global fit than alternatives, even when the other models were themselves used to simulate data $(33,38-46)$. For example, skewed item distributions and unmodeled cross-loadings or correlated residuals can all lead fit statistics to favor the bifactor model over a correlated-factors model (with no general factor), even if the correlatedfactors model more accurately describes the true structure $(46,47)$. The bifactor model's flexibility can also result in good model-data fit even when used with very noisy data or nonsense response patterns $(33,45)$. Thus, it is inappropriate to use global fit statistics to evaluate the bifactor model or favor it over

\footnotetext{
${ }^{4} \mathrm{~A}$ further challenge to using global model fit to compare models is that the bifactor model and many alternatives (e.g., correlated-factors, higher-order) make very similar predictions about observed item covariances, so global fits of all these models are likely to be similar $(47,49)$.
}

alternative models $(29,48) .{ }^{4}$ Instead, choosing to apply the bifactor model should be based on the specific research question. In applying the bifactor model, we suggest that researchers adopt the stance "all models are wrong but some are useful" $(50,51)$. Below, we describe three types of research questions bifactor models are well-suited to address.

\section{Useful Applications of the Bifactor Model}

When a latent variable model is fit to psychopathology data (see 52,53 for discussions on choice of latent variable models versus alternatives, such as network models), bifactor models are useful for their ability to separate indicator variance associated with a general factor from variance associated with narrower group factors or specific indicators. This separation of general from unique variance can inform several questions.

Presence, Strength, and Content of a General Factor. The most immediate question bifactor modeling can address is: if a general factor is present, how strong is it and what content characterizes it? An example of this type of question concerns the widely-noted covariation between major depressive disorder and anxiety disorders. The tripartite model of anxiety-depression co-occurrence posits a common core of general distress, physiological hyperarousal, and anhedonia (54). Simms et al. (55) used a bifactor model and found that all indicators loaded similarly and strongly onto the general factor (along with group anxiety and depression factors), supporting that depression and anxiety share a common core interpreted as "general distress".

Questions concerning strength and content of a general factor should focus on the pattern of factor loadings. What content makes up the general factor? Is it even across indicators or dominated by just a few? Are factor loadings strong or weak? For example, loadings of 
specific cognitive ability tests show very strong loadings (.50-.70) on the general cognitive ability factor (25) (cf. Figure 1A), suggesting it is a major factor that must be explained understand cognitive test performance. Conversely, loadings of Big Five scale scores onto the general factor of self-reported normalrange personality are weaker and more variable (e.g., mean $\lambda \square=\square .27$, range .12-.49) (26), suggesting this factor is not really a "Big One" personality factor (56). At the extreme, the results in Figure 1B for a measure of social attitudes suggest no general factor at all. Here, the general factor only reflects indicators of one group factor, with negligible loadings for other indicators. This pattern suggests that a general factor should be rejected entirely for these scales.

Relatedly, bifactor analysis can help to elucidate the content of group factors. Are indicator loadings onto a group factor meaningfully large and in the theoretically expected direction (cf. Figure 1A)? Or does the group factor mostly reflect idiosyncratic features of only a few indicators (cf. $F_{C}$ in Figure 1B) or an uninterpretable pattern of positive and negative loadings? The latter patterns would imply that there is not a coherent group factor separable from the content contained in the general factor. For discussions of best practices in interpreting patterns of factor loadings in bifactor models, see (10,34,57). To enhance comparability across samples, researchers should consider drawing on previous bifactor model results to add additional constraints to the model, such as constraining the relative magnitudes of indicator loadings on general versus group factors, using informative Bayesian priors based on previous studies, or even fixing factor loadings to specific previously estimated values.
Caveat-Indicator selection influences general factor meaning. Like any model, results of the bifactor model are influenced by the indicators included. The exact meaning of the general factor can change depending on the measures included in the analyses. Results of psychopathology bifactor analyses have been inconsistent due to variations in the indicators used. For example, the $p$-factor has variously reflected general distress $(11,12,36,58)$, psychosis (7), uni/bipolar depression (7), and self and interpersonal dysfunction (59-61).

If indicators from one subgroup are overrepresented, these may come to dominate the general factor. For example, in psychopathology bifactor analyses, internalizing indicators are often overrepresented $(11,13)$, leading the $p$-factor to primarily reflect these features. Specific uncommonly modeled indicators can also substantially alter patterns of factor loadings if included or excluded. For example, including borderline personality disorder can strengthen all loadings on the $p$ factor due to BPD's moderate correlations with most other DSM diagnoses (60). Including eating disorders or specific phobia can clarify the distinction between the $p$-factor and the internalizing group factor (12).

Indicator level of specificity also influences bifactor model results. Bifactor models in psychopathology research have been fit to individual self-report or clinician-ascertained symptoms $(55,62)$, self- or informant-reported scale sum scores $(11,48,63)$, clinician-ascertained symptom counts $(7,13,14)$, and DSMbased categorical diagnoses $(22,36)$. For example, common criteria or symptom overlap across DSM-based disorders may inflate the strength of the general factor if modeled using symptom counts or diagnoses rather than individual symptoms unless steps are taken to correct for overlap (64). These concerns are also relevant if modeling 
relationships among scale sum scores, as many scales include similar items related to general distress or emotional lability. Similarly, if skip-out logic with zero-imputation is used during assessment, this may also inflate correlations among diagnosis indicators and, accordingly, the strength of the general factor.

\section{Caveat-Sampling can impact general} factor strength and content. The sample used can also impact the strength and pattern of loadings onto the general and group factors-though this issue is not specific to bifactor models. For example, university students are less likely to report multimorbidity than inpatient-clinical samples, leading to a weaker general factor. Some clinical instruments, such as the Hamilton Depression Rating Scale (65), may also have low precision in relatively healthy student or community samples, reducing reliability and general factor loadings. In an inpatient clinical or otherwise distressed/help-seeking sample, respondents may have high levels of acute distress and endorse a wide variety of negative symptoms. This will increase loadings onto the general factor and change its interpretation to more reflect current distress than persistent psychopathology (66). Comingling samples with large mean differences (e.g., college students and clinical sample) might produce a spurious latent taxon, especially when the indicators are truly present or absent, rather than dimensional (e.g., hallucinations). Features of certain disorders, such as BPD or depression, might similarly increase acquiescent endorsement of negative symptoms. This will increase $p$-factor strength if these disorders are frequently represented in a sample.

These more artefactual forms of a general factor can be addressed by using stronger research designs than cross-sectional selfratings. For example, modeling a stable general factor in longitudinal data can help to disentangle persistent general psychopathology from current distress (67). Influences of distress-acquiescent responding can be reduced by using multi-rater or multi-method designs (67). In non-self-rating designs, there is little evidence for a general factor of normalrange personality (27); in informant-ratings or across raters, only two weakly correlated higher-

order metatraits- $\alpha /$ Integration/Stability and $\beta /$ Exploration/Plasticity-are supported (68). This finding suggests that the general factor of normal-range personality is a self-ratingspecific evaluative factor. In contrast, parentratings and multi-rater-ratings of psychopathology tend to show a similarly strong general $p$-factor as self-ratings, though cf. (69).

Reliability Analysis to Guide Application and Interpretation of Scales. A second useful application of bifactor analyses is reliability analysis to guide interpretations of total versus subtest scores from multifaceted inventories. Using factor loadings from a bifactor analysis, researchers can compute $\omega_{h}$ (omega hierarchical) reliability, which denotes the proportion of variance in a total sum score attributable to the general factor (70-73). If $\omega_{h}$ is high, as in cognitive ability batteries (74), this supports computing a total score and interpreting it as reflecting primarily the general factor. If $\omega_{h}$ is small, as in self-rated normal-range personality $(26,56)$, this indicates that a total score is better understood as a composite of narrower factors; researchers should consider whether computing a total score or focusing on subtest scores is more meaningful. For example, several psychometric studies of the Anxiety Sensitivity Index (75) have examined the utility of its three subscales. These studies have found that the anxiety general factor accounted for over $75 \%$ of the variance in the items, whereas the subscale group factors generally accounted 
for negligible amounts of variance $(76,77)$. Even more dramatically, bifactor analyses of the Derogatis Symptom Checklist and Brief Symptom Inventory $(78,79)$ have found that the general factor accounts for $\approx 95 \%$ of the variance in these items $(80,81)$. These results suggest that these measures should primarily be interpreted using total scores. Depending on the sampling and research design (e.g., see above), we may even question whether the narrow constructs captured by the group factors are empirically distinguishable constructs.

Relatedly, for each subscale, researchers can compute $\omega_{s}$ (omega subscale), the reliability of the subscale after removing variance associated with the general factor. If $\omega_{h}$ is large but $\omega_{s}$ is small, then the remaining variance in the specific factors may not be meaningfully interpretable, and any individual differences cannot be reliably captured in narrower facets separate from the general factor. For example, the Wechsler Adult Intelligence Scale subscales have weak reliability after controlling for general cognitive ability $\left(\omega_{s} \square=\square .13-.47\right)$ (74). Similarly, on average across psychopathology measures, only $\approx 37 \%$ of the variance ( $43 \%$ of the re liable variance [general $\square+\square$ group factors, excluding item-specific variance and error]) in subscales was attributable to the narrow group factor constructs (69). These results indicate that many psychopathology subscales should primarily be interpreted as reflecting the general factor; interpreting differential subscale profiles may be highly unreliable (see also 82).

Relations of General and Group Factors with External Variables. A third useful application of bifactor analysis is examining differential relations of general and narrow group factors with external variables, such as correlations with putative antecedents, criterion variables, or changes in response to treatment. For example, in parent-rated child psychopathology, low executive functioning and family relationship risk factors were related to the general $p$-factor, but not to fear, distress, or externalizing group factors (13). In contrast, both the $p$-factor and externalizing group factor prospectively predicted teacherreported academic performance, behavioral problems, grade retention, and special education status (11). In adults, both the $p$-factor and internalizing group factor uniquely predicted suicidality and non-suicidal selfinjury (15).

Because observed total scores or subscale scores may reflect a mixture of general and group factor variance, observed score correlations might reflect the influence of the general factor, the group factor, or both. Observed correlations may be inflated or attenuated as estimates of relations between the broad/narrow psychopathology constructs and other variables (83). For example, the perfectionism facet of Conscientiousness was negatively related to university student physical and mental health after controlling for general Conscientiousness; this relationship was obscured when both sources of variance were combined in the observed subscale score (84). By separating the predictive power of broad and narrow factors, bifactor modeling can provide a clearer picture of the nomological network of psychopathology.

Elaborating the stability and nomological network of general and group factors is also useful for discerning whether factors reflect substantive constructs or artefacts (85). For example, studies of childhood and adolescent psychopathology have found that both the $p$ factor and group factors are stable and similar in factor strength over time, suggesting that the $p$-factor reflects more than transient current-distress $(14,67,86,87)$. Similarly, if a general factor shows unique relationships 
with important antecedents, biological substrates, or outcomes, this supports its interpretation as a meaningful construct; the $p$-factor shows genetic correlations with Neuroticism (12) and reduced gray matter volume in prefrontal areas (19).

Practically, bifactor analyses aimed at assessing the reliability and external validity of the general and group factors can help guide the level of focus in assessment and treatment planning $(17,88,89)$. Can we predict treatment outcomes based on the general factor alone, or do we need an assessment of group factors? Does matching patients to treatment based on narrow group factors produce better outcomes than matching them on the general factor alone? Do broad-based treatments like dialectical behavior therapy target only general psychopathology or do they also affect narrow personality disorder aspects as well?

\section{Comparison with Alternative Models}

We describe several useful applications of bifactor models. This is not to suggest that they are a panacea or appropriate for all research questions. Below, we compare the bifactor model to several common alternatives and consider when these alternatives may be more useful.

Higher-Order Model. The most similar model to the bifactor is the higher-order factor model, where the items load on their respective group factors which then load on the general factor. Researchers sometimes prefer to use a higher-order model if their theoretical model conceptualizes the narrow factors as components of the general factor, rather than distinct entities. For example, one common liabilities model posits that individuals first develop a general liability for psychopathology, which then differentiates into more specific pathologies in response to environmental experiences (90).

However, even though the higher-order model may on its face appear more appropriate for such conceptualizations, mathematically it is extremely similar to the bifactor model. Indeed, the higher-order model is simply a somewhat more constrained version of the bifactor model $(23,42,47)$. Given their mathematical similarity, the two models make very similar predictions about indicator covariances and typically yield comparable results. Hypotheses that can be tested using higher-order model can generally be more easily tested using the bifactor model. The advantage of the bifactor model for describing a general factor over the higher-order model is that it directly teases apart the unique contributions to the indicators of the general and group factors (25). In the bifactor model, unique aspects of the group factors are represented as distinct variables from the general factor. In the higher-order model, unique aspects of the group factors are represented as the residuals (disturbances) of the first-order latent variables which together with variance from the general factor jointly influence the indicators. This arrangement makes it more difficult to use reliability analysis to assess the degree to which scores primarily reflect the general or group factors. Likewise, it becomes more difficult to assess the differential external validity of the general or group factors since the unique relations of the group factors must be drawn from the residuals (disturbances), rather than the latent group factors themselves. To increase clarity, even when a higher-order model is theoretically preferred, results are often presented in the form of a constrained bifactor model using the SchmidLeiman transformation (34). In general, we argue that the bifactor model makes testing theoretical hypotheses about general and group factors clearer and more interpretable, 
even if the conceptual model posits that the general factor directly contributes to the narrow group factors.

Correlated Traits Model. The correlated traits model includes correlated group factors but no overarching general factor-e.g., a model specifying internalizing and externalizing factors with no general $p$-factor (2). If correlations among first-order factors are small, the correlated traits model can provide a simpler and easier-to-interpret description of the structure of a measure. However, if correlations among factors are large, the bifactor model can be more useful, particularly for discerning whether factors' common or unique variance is the primary source of measures' predictive power; cf. the challenges of estimating relative importance for correlated predictors (91-93).

Network Models. A more recently developed psychometric approach, network models posit that indicators (e.g., symptoms) directly influence each other without any unobserved latent variables (94). These models are used to test dynamic mutualism and other network theories of psychopathology, which hypothesize that covariance among symptoms does not reflect a common latent variable, but rather the effects of individual symptoms reinforcing each other (e.g., a cascading downward spiral) $(95,96)$; the $p$ factor is an effect of symptom covariance, not a cause (97). Although network versus common cause theories of psychopathology are importantly distinct, it is important to remember that the mathematics of common factor and network models are highly similar $(98,53,52)$. Every network model can be expressed as an equivalent factor model, and vice versa. Cross-sectional network models have the same limitations as factor models in terms of interpreting the meaning of symptom co-occurrence; network models themselves cannot provide insight into processes or development any more than common factor analysis can. Where network models can be useful compared to the bifactor (or other common factor models) is if the research question concerns the nomological network of individual symptoms. For example, Fried et al. (99) used network analysis to examine unique relationships of individual depression symptoms with inflammation biomarkers, cf. $(100,101)$. Because indicator-specific variance is typically regarded as error in bifactor and other common factor models, such symptom-specific relationships are easier to examine with network models. As with factor models, exploring processes with network models requires longitudinal data.

\section{The Bifactor Model and Biological Substrates of Psychopathology}

A growing area of research examines biological substrates of psychological constructs, such as neurobiological and genetic correlates of individual differences in personality, cognition, or psychopathology (102-105). For example, several studies have examined or proposed correlations of psychopathology general and group factors with genetic singlenucleotide-polymorphisms or neurobiological variables (e.g., gray matter volume; volume or activation of amygdala/PFC circuits, HPA axis, hippocampus, ventral striatum) $(19,106-$ 109). These questions may benefit from bifactor models' utility for examining external variable relations. For example, if individual symptoms or disorders show stronger or more coherent associations with genetic, neuro-biological, or biomarker variables than with latent factors, these associations might suggest that the latent factors do not reflect specific biological liabilities but rather reflect measurement artefacts or common socioenvironmental factors. Conversely, if many 
symptoms are associated with common biological substrates, and extracted latent variables show even stronger relations with these substrates, this would support an interpretation of common biological liabilities for diverse psychopathology indicators. In this way, neurobiological or genetic data can serve as a constraint that can help to discriminate between alternative models that psychological data alone cannot differentiate, cf. (110).

Careful attention must be paid, however, to measurement fidelity of biological variables. For example, functional neuroimaging is notoriously plagued by artefacts and instability of extracted intrinsic connectivity networks (111). Similarly, factor analyses of allele co-occurrence have dubious interpretation. Because of humans' small effective population size, compared to other species, natural selection effects in humans are frequently smaller than genetic drift effects, cf. (112). Accordingly, co-occurrence of specific alleles in a population does not necessarily reflect a consequence of selection or otherwise indicate genetic substrates for a specific complex phenotype (such as psychopathology). Remedies such as removing the first principal component cannot completely remove these effects and may lose relevant genetic information. Likewise, fitting latent variable models-including bifactor models-to polygenic risk scores might suggest shared genetic correlates, but this does not imply the $p$-factor is a unitary biological entity or alone reveal biological mechanisms; genes code for proteins, not psychopathology (113). These issues can make it challenging to interpret studies that fit factor models to both psychopathology measures and genetic or neurobiological variables (114-117). In modeling neurobiological or genetic data, researchers must first ensure that their models of neural or genetic structure are consistent with underlying molecular processes.
Ultimately, an important point to remember is that studying biological substrates does not make phenomena being studied more "real" or valid. Psychopathology is phenomenologically defined and diagnosed at the level of affective, cognitive, and behavioral symptoms; biological substrates may be important, but they are not necessary to understand assessment, diagnosis, development, or treatment of psychological disorders (118, 119). Psychological and biological explanations complement each other; they reflect different levels of specificity to explain the same phenomena, but there is no one-to-one mapping between psychological constructs and biological factors. Instead, psychological constructs emerge from interactions among dynamic processes over development (105).

\section{Modeling Cannot Fix Inadequate Research Design}

To close, we reiterate that statistical modeling cannot make fundamental limitations of data disappear. The questions that data can address are a function of the research design, not the model chosen to analyze them. Crosssectional relationships among indicators cannot speak to developmental processes, regardless of the type of model [bifactor, network] or indicator [behavioral symptoms, biological variables] used. The appropriate level of analysis for psychopathology (e.g., symptoms, disorders, spectra, $p$-factor) is a question best addressed empirically-e.g., by comparing genetic and neurobiological correlates of individual symptoms versus broader factors $(31,109)$-or pragmatically-e.g., do components from one model yield stronger predictions of clinically-relevant outcomes than components from another model? Paired with rigorous research designs that can rule out methodological confounds, such as longitudinal and multi-informant designs, bifactor modeling can be a useful tool for 
investigating the nature of psychopathology constructs (broad and narrow) and their mechanisms, development, and responsiveness to treatment.

\section{Acknowledgements and Disclosures}

This work was supported by DA032582 (NIDA).

The authors report no biomedical financial interests or potential conflicts of interest.

\section{Article Information}

From the Department of Psychology, University of South Florida, and the Department of Cell Biology, Microbiology and Molecular Biology, University of South Florida.

Address correspondence to Marina A. Bornovalova or Brenton M. Wiernik, Department of Psychology, University of South Florida, 4202 East Fowler Ave, PCD4118G, Tampa, FL 33620. Email: bornovalova@usf.edu or brenton@wiernik.org

\section{References}

1. Newman DL, Moffitt TE, Caspi A, Silva PA (1998): Comorbid mental disorders: implications for treatment and sample selection. J Abnorm Psychol 107: 305-311.

2. Krueger RF (1999): The structure of common mental disorders. Arch Gen Psychiatry 56: 921.

3. Krueger RF, Markon KE (2006): Reinterpreting comorbidity: a model-based approach to understanding and classifying psychopathology. Annu Rev Clin Psychol 2: 111-133.

4. Neale MC, Kendler KS (1995): Models of comorbidity for multifactorial disorders. Am J Hum Genet 57: 935-953.

5. Reise SP (2012): The rediscovery of bifactor measurement models. Multivar Behav Res 47: 667-696.

6. Holzinger KJ, Swineford F (1937): The bifactor method. Psychometrika 2: 41-54.

7. Caspi A, Houts RM, Belsky DW, Goldman-Mellor SJ, Harrington H, Israel S, et al. (2014): The p factor: one general psychopathology factor in the structure of psychiatric disorders? Clin Psychol Sci 2: 119137.

8. Heinrich $M$, Zagorscak $P$, Eid $M$, Knaevelsrud $C$ (2018): Giving $G$ a meaning: an application of the bifactor-(S-1) approach to realize a more symptomoriented modeling of the Beck Depression Inventory-II. Assessment. https://doi.org/10/gfdshb

9. Burns GL, Geiser C, Servera M, Becker SP, Beauchaine TP (2019): Application of the bifactor S - 1 model to multisource ratings of ADHD/ODD symptoms: an appropriate bifactor model for symptom ratings. $J$ Abnorm Child Psychol. https://doi.org/10/gghwjs

10. Markon KE (2019): Bifactor and hierarchical models: specification, inference, and interpretation. Annu Rev Clin Psychol 15: 51-69.

11. Lahey BB, Rathouz PJ, Keenan K, Stepp SD, Loeber R, Hipwell AE (2015): Criterion validity of the general factor of psychopathology in a prospective study of girls. J Child Psychol Psychiatry 56: 415422.

12. Tackett JL, Lahey BB, van Hulle C, Waldman I, Krueger RF, Rathouz PJ (2013): Common genetic influences on negative emotionality and a general psychopathology factor in childhood and adolescence. J Abnorm Psychol 122: 1142-1153.

13. Martel MM, Pan PM, Hoffmann MS, Gadelha A, do Rosário MC, Mari JJ, et al. (2017): A general psychopathology factor ( $P$ factor) in children: structural model analysis and external validation through familial risk and child global executive function. J Abnorm Psychol 126: 137-148.

14. Castellanos-Ryan N, Brière FN, O'Leary-Barrett $M$, Banaschewski T, Bokde A, Bromberg $\mathrm{U}$, et al. (2016): The structure of psychopathology in adolescence and its common personality and cognitive correlates. J Abnorm Psychol 125: 1039-1052.

15. Haltigan JD, Aitken M, Skilling T, Henderson J, Hawke L, Battaglia M, et al. (2018): "P" and "DP": examining symptom-level bifactor models of psychopathology and dysregulation in clinically referred children and adolescents. J Am Acad Child Adolesc Psychiatry 57: 384-396.

16. Pettersson $\mathrm{E}$, Lahey $\mathrm{BB}$, Larsson $\mathrm{H}$, Lichtenstein $\mathrm{P}$ (2018): Criterion validity and utility of the general factor of psychopathology in childhood: predictive associations with independently measured severe adverse mental health outcomes in adolescence. J Am Acad Child Adolesc Psychiatry 57: 372-383.

17. Forbes MK, Rapee RM, Krueger RF (2019): Opportunities for the prevention of mental disorders by reducing general psychopathology in early childhood. Behav Res Ther 119: 103411. 
18. Eaton NR, Krueger RF, Keyes KM, Wall M, Hasin DS, Markon KE, et al. (2013): The Structure and Predictive Validity of the Internalizing Disorders. $J$ Abnorm Psychol 122: 86-92.

19. Snyder HR, Hankin BL, Sandman CA, Head K, Davis EP (2017): Distinct patterns of reduced prefrontal and limbic gray matter volume in childhood general and internalizing psychopathology. Clin Psychol Sci 5: 1001-1013.

20. Kim H, Eaton NR (2015): The hierarchical structure of common mental disorders: connecting multiple levels of comorbidity, bifactor models, and predictive validity. J Abnorm Psychol 124: 1064-1078.

21. Lahey BB, Krueger RF, Rathouz PJ, Waldman ID, Zald DH (2017): A hierarchical causal taxonomy of psychopathology across the life span. Psychol Bull 143: 142-186.

22. Lahey BB, Applegate B, Hakes JK, Zald DH, Hariri AR, Rathouz PJ (2012): Is there a general factor of prevalent psychopathology during adulthood? J Abnorm Psychol 121: 971-977.

23. Yung Y-F, Thissen D, McLeod LD (1999): On the relationship between the higher-order factor model and the hierarchical factor model. Psychometrika 64: 113-128.

24. Kotov R, Krueger RF, Watson D, Achenbach TM, Althoff RR, Bagby RM, et al. (2017): The Hierarchical Taxonomy of Psychopathology (HiTOP): a dimensional alternative to traditional nosologies. $J A b$ norm Psychol 126: 454-477.

25. Cucina J, Byle K (2017): The bifactor model fits better than the higher-order model in more than $90 \%$ of comparisons for mental abilities test batteries. $J$ Intell 5: 27.

26. Davies SE, Connelly BL, Ones DS, Birkland AS (2015): The general factor of personality: the "Big One," a self-evaluative trait, or a methodological gnat that won't go away? Personal Individ Differ 81: 13-22.

27. Chang L, Connelly BS, Geeza AA (2012): Separating method factors and higher order traits of the Big Five: a meta-analytic multitrait-multimethod approach. J Pers Soc Psychol 102: 408-426.

28. Wiernik BM (2016, December): The Nomological Network of Classic and Contemporary Career Preferences. Minneapolis, MN: University of Minnesota. Retrieved from https://hdl.handle.net/11299/202184

29. Bonifay W, Lane SP, Reise SP (2017): Three concerns with applying a bifactor model as a structure of psychopathology. Clin Psychol Sci 5: 184-186.

30. Eid M, Geiser C, Koch T, Heene M (2017): Anomalous results in G-factor models: Explanations and alternatives. Psychol Methods 22: 541-562.
31. van Bork R, Epskamp S, Rhemtulla M, Borsboom D, van der Maas HLJ (2017): What is the p-factor of psychopathology? Some risks of general factor modeling. Theory Psychol 27: 759-773.

32. Eid M, Krumm S, Koch T, Schulze J (2018): Bifactor Models for Predicting Criteria by General and Specific Factors: Problems of Nonidentifiability and Alternative Solutions. J Intell 6: 42.

33. Bonifay W, Cai L (2017): On the complexity of item response theory models. Multivar Behav Res 52: 465-484.

34. Giordano CA, Waller NG (2019): Recovering bifactor models: a comparison of seven methods. Psychol Methods. https://doi.org/10.1037/met0000227

35. Lahey BB, Zald DH, Perkins SF, Villalta-Gil V, Werts KB, Hulle CAV, et al. (2018): Measuring the hierarchical general factor model of psychopathology in young adults. Int J Methods Psychiatr Res 27: e1593.

36. Greene AL, Eaton NR (2017): The temporal stability of the bifactor model of comorbidity: an examination of moderated continuity pathways. Compr Psychiatry 72: 74-82.

37. Hyland $P$, Murphy J, Shevlin M, Carey S, Vallières F, Murphy D, Elklit A (2018): Correlates of a general psychopathology factor in a clinical sample of childhood sexual abuse survivors. J Affect Disord 232: 109-115.

38. Gignac GE (2016): The higher-order model imposes a proportionality constraint: that is why the bifactor model tends to fit better. Intelligence 55: 57-68.

39. Mansolf M, Reise SP (2016): Exploratory bifactor analysis: the Schmid-Leiman orthogonalization and Jennrich-Bentler analytic rotations. Multivar Behav Res 51: 698-717.

40. Maydeu-Olivares A, Coffman DL (2006): Random intercept item factor analysis. Psychol Methods 11: 344-362.

41. McFarland DJ (2016): Modeling general and specific abilities: evaluation of bifactor models for the WJ-III. Assessment 23: 698-706.

42. Molenaar D (2016): On the distortion of model fit in comparing the bifactor model and the higher-order factor model. Intelligence 57: 60-63.

43. Morgan G, Hodge K, Wells K, Watkins M (2015): Are fit indices biased in favor of bifactor models in cognitive ability research? A comparison of fit in correlated factors, higher-order, and bifactor models via Monte Carlo simulations. J Intell 3: 2-20.

44. Murray AL, Johnson W (2013): The limitations of model fit in comparing the bi-factor versus higher-order models of human cognitive ability structure. Intelligence 41: 407-422. 
45. Reise SP, Kim DS, Mansolf M, Widaman KF (2016): Is the bifactor model a better model or is it just better at modeling implausible responses? Application of iteratively reweighted least squares to the Rosenberg Self-Esteem Scale. Multivar Behav Res 51: 818-838.

46. Greene AL, Eaton NR, Li K, Forbes MK, Krueger RF, Markon KE, et al. (2019): Are fit indices used to test psychopathology structure biased? A simulation study. J Abnorm Psychol. https://doi.org/10/gf5krm

47. Mansolf M, Reise SP (2017): When and why the second-order and bifactor models are distinguishable. Intelligence 61: 120-129.

48. Watts AL, Poore HE, Waldman ID (2019): Riskier tests of the validity of the bifactor model of psychopathology [Advance online publication]. Clin Psychol Sci. https://doi.org/10/gf6bhr

49. Credé M, Harms PD (2015): 25 years of higher-order confirmatory factor analysis in the organizational sciences: A critical review and development of reporting recommendations. J Organ Behav 36: 845872.

50. Box GEP (1979): Robustness in the strategy of scientific model building. In: Launer RL, Wilkinson GN, editors. Robustness in Statistics. Academic Press, pp 201-236.

51. Snyder HR, Hankin BL (2017): All models are wrong, but the $\mathrm{p}$ factor model is useful: reply to Widiger and Oltmanns (2017) and Bonifay, Lane, and Reise (2017). Clin Psychol Sci 5: 187-189.

52. Hallquist MN, Wright AGC, Molenaar PCM (2019): Problems with centrality measures in psychopathology symptom networks: why network psychometrics cannot escape psychometric theory. Multivar Behav Res. https://doi.org/10/gf6jwg

53. Epskamp S, Maris G, Waldorp LJ, Borsboom D (2018): Network psychometrics. In: Irwing P, Booth T, Hughes DJ, editors. The Wiley Handbook of Psychometric Testing. Chichester, UK: Wiley, pp 953986.

54. Clark LA, Watson D (1991): Tripartite model of anxiety and depression: psychometric evidence and taxonomic implications. J Abnorm Psychol 100: 316-336.

55. Simms LJ, Grös DF, Watson D, O'Hara MW (2008): Parsing the general and specific components of depression and anxiety with bifactor modeling. Depress Anxiety 25: E34-E46.

56. Revelle W, Wilt J (2013): The general factor of personality: a general critique. J Res Personal 47: 493504.
57. Chen FF, West SG, Sousa KH (2006): A comparison of bifactor and second-order models of quality of life. Multivar Behav Res 41: 189-225.

58. Lahey BB, Van Hulle CA, Singh AL, Waldman ID, Rathouz PJ (2011): Higher-order genetic and environmental structure of prevalent forms of child and adolescent psychopathology. Arch Gen Psychiatry 68: 181-189.

59. Jahng S, Trull TJ, Wood PK, Tragesser SL, Tomko $\mathrm{R}$, Grant JD, et al. (2011): Distinguishing general and specific personality disorder features and implications for substance dependence comorbidity. $J$ Abnorm Psychol 120: 656-669.

60. Sharp C, Wright AGC, Fowler JC, Frueh BC, Allen JG, Oldham J, Clark LA (2015): The structure of personality pathology: both general (' $g$ ') and specific ('s') factors? J Abnorm Psychol 124: 387-398.

61. Wright AGC, Hopwood CJ, Skodol AE, Morey LC (2016): Longitudinal validation of general and specific structural features of personality pathology. $J$ Abnorm Psychol 125: 1120-1134.

62. Patalay P, Fonagy P, Deighton J, Belsky J, Vostanis $P$, Wolpert M (2015): A general psychopathology factor in early adolescence. Br J Psychiatry 207: 1522.

63. Laceulle OM, Vollebergh WAM, Ormel J (2015): The structure of psychopathology in adolescence: replication of a general psychopathology factor in the TRAILS Study. Clin Psychol Sci 3: 850-860.

64. Borsboom D (2002): The structure of the DSM. Arch Gen Psychiatry 59: 569-570.

65. Hamilton M (1960): A rating scale for depression. J Neurol Neurosurg Psychiatry 23: 56-62.

66. Böhnke JR, Lutz W, Delgadillo J (2014): Negative affectivity as a transdiagnostic factor in patients with common mental disorders. J Affect Disord 166: 270278.

67. Snyder HR, Young JF, Hankin BL (2017): Strong homotypic continuity in common psychopathology-, internalizing-, and externalizing-specific factors over time in adolescents. Clin Psychol Sci 5: 98-110.

68. Stanek KC, Ones DS (2018): Taxonomies and compendia of cognitive ability and personality measures relevant to industrial, work, and organizational psychology. In: Ones DS, Anderson N, Viswesvaran C, Sinangil HK, editors. The SAGE Handbook of Industrial, Work and Organizational Psychology, 2nd ed., vol. 1. London, United Kingdom: Sage, pp 366-407.

69. Constantinou M, Fonagy P (2019, August 6): Evaluating bifactor models of psychopathology using model-based reliability indices. PsyArXiv. https://doi.org/10/gf6jwj 
70. McDonald RP (2013): Test Theory: A Unified Treatment, 1st ed. Psychology Press. https://doi.org/10.4324/9781410601087

71. Zinbarg RE, Barlow DH, Brown TA (1997): Hierarchical structure and general factor saturation of the Anxiety Sensitivity Index: evidence and implications. Psychol Assess 9: 277-284.

72. Rodriguez A, Reise SP, Haviland MG (2016): Evaluating bifactor models: calculating and interpreting statistical indices. Psychol Methods 21: 137-150.

73. Zinbarg RE, Yovel I, Revelle W, McDonald RP (2006): Estimating generalizability to a latent variable common to all of a scale's indicators: a comparison of estimators for Wh. Appl Psychol Meas 30: 121-144.

74. Gignac GE, Watkins MW (2013): Bifactor modeling and the estimation of model-based reliability in the WAIS-IV. Multivar Behav Res 48: 639-662.

75. Reiss S, Peterson RA, Gursky DM, McNally RJ (n.d.): Anxiety sensitivity, anxiety frequency and the prediction of fearfulness. Behav Res Ther Vol 24 Issue 11986 Pages 1-8 24: 1-8.

76. Ebesutani C, McLeish AC, Luberto CM, Young J, Maack DJ (2014): A bifactor model of anxiety sensitivity: analysis of the Anxiety Sensitivity Index-3. J Psychopathol Behav Assess 36: 452-464.

77. Osman A, Gutierrez PM, Smith K, Fang Q, Lozano G, Devine A (2010): The Anxiety Sensitivity Index3: analyses of dimensions, reliability estimates, and correlates in nonclinical samples. J Pers Assess 92: 45-52.

78. Derogatis LR (2017): Symptom Checklist-90-Revised, Brief Symptom Inventory, and BSI-18. In: Maruish ME, editor. Handbook of Psychological Assessment in Primary Care Settings. New York, NY, USA: Routledge, pp 599-630.

79. Derogatis LR, Cleary PA (1977): Confirmation of the dimensional structure of the SCL-90: a study in construct validation. J Clin Psychol 33: 981-989.

80. Lu Y, Alvarez AN, Miller MJ (2019): Measurement invariance of the Brief Symptom Inventory-18 (BSI18) across Asian American ethnic, nativity, and gender groups. Asian Am J Psychol 10: 1-10.

81. Urbán R, Kun B, Farkas J, Paksi B, Kökönyei G, Unoka Z, et al. (2014): Bifactor structural model of symptom checklists: SCL-90-R and Brief Symptom Inventory (BSI) in a non-clinical community sample. Psychiatry Res 216: 146-154.

82. Bulut O, Davison ML, Rodriguez MC (2017): Estimating between-person and within-person subscore reliability with profile analysis. Multivar Behav Res 52: 86-104.
83. Wiernik BM, Wilmot MP, Kostal JW (2015): How data analysis can dominate interpretations of dominant general factors. Ind Organ Psychol 8: 438-445.

84. McAbee ST, Oswald FL, Connelly BS (2014): Bifactor models of personality and college student performance: a broad versus narrow view. Eur J Personal 28: 604-619.

85. Hankin BL (2019): A choose your own adventure story: Conceptualizing depression in children and adolescents from traditional DSM and alternative latent dimensional approaches. Behav Res Ther 118: 94-100.

86. Murray AL, Eisner M, Ribeaud D (2016): The development of the general factor of psychopathology ' $p$ factor' through childhood and adolescence. J Abnorm Child Psychol 44: 1573-1586.

87. McElroy E, Belsky J, Carragher N, Fearon P, Patalay $P$ (2018): Developmental stability of general and specific factors of psychopathology from early childhood to adolescence: dynamic mutualism or p-differentiation? J Child Psychol Psychiatry 59: 667-675.

88. Meier MA, Meier MH (2018): Clinical implications of a general psychopathology factor: a cognitive-behavioral transdiagnostic group treatment for community mental health. J Psychother Integr 28: 253-268.

89. Olino TM, McMakin DL, Forbes EE (2018): Toward an empirical multidimensional structure of anhedonia, reward sensitivity, and positive emotionality: an exploratory factor analytic study. Assessment 25: 679-690.

90. Tully EC, lacono W (2014): An integrative common liabilities model for the comorbidity of substance use disorders with externalizing and internalizing disorders. In: Sher KJ, editor. The Oxford Handbook of Substance Use and Substance Use Disorders, vol. 2. Oxford University Press, pp 187-212.

91. Waller NG (2008): Fungible weights in multiple regression. Psychometrika 73: 691-703.

92. Azen R, Budescu DV (2003): The dominance analysis approach for comparing predictors in multiple regression. Psychol Methods 8: 129-148.

93. Braun MT, Converse PD, Oswald FL (2019): The accuracy of dominance analysis as a metric to assess relative importance: the joint impact of sampling error variance and measurement unreliability. J Appl Psychol 104: 593-602.

94. Borsboom D, Cramer AOJ (2013): Network analysis: an integrative approach to the structure of psychopathology. Annu Rev Clin Psychol 9: 91-121.

95. Fried El, van Borkulo CD, Cramer AOJ, Boschloo L, Schoevers RA, Borsboom D (2017): Mental disorders as networks of problems: a review of recent insights. Soc Psychiatry Psychiatr Epidemiol 52: 1-10. 
96. Borsboom D (2017): A network theory of mental disorders. World Psychiatry 16: 5-13.

97. Van Der Maas HLJ, Dolan CV, Grasman RPPP, Wicherts JM, Huizenga HM, Raijmakers MEJ (2006): A dynamical model of general intelligence: The positive manifold of intelligence by mutualism. Psychol Rev 113: 842-861.

98. Molenaar PCM (2003): State space techniques in structural equation modeling: transformation of latent variables in and out of latent variable models [Unpublished manuscript]. Retrieved August 16, 2019, from https://quantdev.ssri.psu.edu/resources/state-space-techniques-structural-equation-modeling-transformation-latent-variables-and

99. Fried El, Haslbeck J (2019, August 13): Using network analysis to examine links between individual depression symptoms, inflammatory markers, and covariates. PsyArXiv. https://doi.org/10/gf6js2

100. Borsboom D, Cramer AOJ, Schmittmann VD, Epskamp S, Waldorp LJ (2011): The small world of psychopathology. PLOS ONE 6: e27407.

101. Cramer AOJ, Kendler KS, Borsboom D (2011): Where are the genes? The implications of a network perspective on gene hunting in psychopathology. Eur J Personal 25: 270-271.

102. DeYoung CG, Grazioplene RG, Allen TA (2019): The neurobiology of personality. In: John OP, Robbins RW, editors. Handbook of Personality: Theory and Research, 4th ed. New York, NY: Guilford Press.

103. Deary IJ, Penke L, Johnson W (2010): The neuroscience of human intelligence differences. Nat Rev Neurosci 11: 201-211.

104. Cuthbert BN (2014): The RDoC framework: facilitating transition from ICD/DSM to dimensional approaches that integrate neuroscience and psychopathology. World Psychiatry 13: 28-35.

105. Beauchaine TP, Zisner A (2017): Motivation, emotion regulation, and the latent structure of psychopathology: An integrative and convergent historical perspective. Int J Psychophysiol 119: 108-118.

106. Shanmugan S, Wolf DH, Calkins ME, Moore TM, Ruparel K, Hopson RD, et al. (2016): Common and Dissociable Mechanisms of Executive System Dysfunction Across Psychiatric Disorders in Youth. Am J Psychiatry 173: 517-526.

107. Grotzinger $A D$, Rhemtulla $M$, de Vlaming R, Ritchie SJ, Mallard TT, Hill WD, et al. (2018, April 21): Genomic SEM provides insights into the multivariate genetic architecture of complex traits. BioRxiv. https://doi.org/10.1101/305029

108. Jones HJ, Heron J, Hammerton G, Stochl J, Jones PB, Cannon M, et al. (2018): Investigating the genetic architecture of general and specific psychopathology in adolescence. Transl Psychiatry 8: 111.

109. Zald DH, Lahey BB (2017): Implications of the hierarchical structure of psychopathology for psychiatric neuroimaging. Biol Psychiatry Cogn Neurosci Neuroimaging 2: 310-317.

110. Marsolek CJ, Burgund ED (2008): Dissociable neural subsystems underlie visual working memory for abstract categories and specific exemplars. Cogn Affect Behav Neurosci 8: 17-24.

111. Poppe AB, Wisner K, Atluri G, Lim KO, Kumar V, MacDonald AW (2013): Toward a neurometric foundation for probabilistic independent component analysis of fMRI data. Cogn Affect Behav Neurosci 13: 641-659.

112. Lynch M (2009): Estimation of allele frequencies from high-coverage genome-sequencing projects. Genetics 182: 295-301.

113. Belsky DW, Harden KP (2019): Phenotypic annotation: using polygenic scores to translate discoveries from genome-wide association studies from the top down. Curr Dir Psychol Sci 28: 82-90.

114. Bolt T, Nomi JS, Yeo BTT, Uddin LQ (2017): Datadriven extraction of a nested model of human brain function. J Neurosci 37: 7263-7277.

115. Luningham JM, Poore HE, Yang J, Waldman ID (2018): Testing structural models of psychopathology at the genomic level. bioRxiv 502039.

116. Allegrini AG, Cheesman R, Rimfeld K, Selzam S, Pingault Jb, Eley TC, Plomin R (2019, March 28): The $p$ factor: genetic analyses support a general dimension of psychopathology in childhood and adolescence. BioRxiv. https://doi.org/10.1101/591354

117. Selzam S, Coleman JRI, Caspi A, Moffitt TE, Plomin $R$ (2018): A polygenic $p$ factor for major psychiatric disorders. Transl Psychiatry 8: 1-9.

118. Ross D, Spurrett D (2004): What to say to a skeptical metaphysician: a defense manual for cognitive and behavioral scientists. Behav Brain Sci 27: 603-627.

119. Gardner C, Kleinman A (2019): Medicine and the mind-the consequences of psychiatry's identity crisis. N Engl J Med 381: 1697-1699. 